\title{
UAV prototype for localization and identification of radioactive contamination and emitters
}

\author{
S.Moretto ${ }^{1,2}$, F.E. Pino Andrades ${ }^{1,3}$, J. Delgado ${ }^{1}$, C.L. Fontana ${ }^{1,2}$, D. Fabris ${ }^{2}$, G. Nebbia ${ }^{2}$, M. Turcato ${ }^{2}$, \\ D. Brunelli ${ }^{4}$, L. Pancheri ${ }^{4}$, A. Quaranta ${ }^{4}$ \\ ${ }^{1}$ Università degli Studi di Padova, via Marzolo 8, 35131, Padova ITALY \\ ${ }^{2}$ Istituto Nazionale di Fisica Nucleare, Sez. Padova, via Marzolo 8, 35131, Padova ITALY \\ ${ }^{3}$ Istituto Nazionale di Fisica Nucleare, Laboratori Nazionali di Legnaro, Viale dell'Università, 2, \\ 35020 Legnaro (Padova) ITALY \\ ${ }^{4}$ Università di Trento, Trento,ITALY \\ Corresponding author: Sandra.moretto@unipd.it
}

\begin{abstract}
The Dragon (Drone for RAdiation detection of Gammas and Neutrons) prototype aims at designing and developing an unmanned aerial vehicle (UAV) equipped with a detection system able to identify radioactive materials, spread over an area or located in a specific position. The system is focused on the localization of the unknown emitter and its subsequently identification.
\end{abstract}

The proposed prototype is made up of two easily interchangeable detection systems, one will be used as a counter while the second will be aimed to perform goodresolution gamma spectroscopy. Both solutions have neutron - gamma discrimination capability in order to be suitable for special nuclear materials (SNM) detection in gamma contamination background.

The data acquisition module is made up of a compact digitizer board (RedPitaya, sampling rate of $125 \mathrm{MHz}$ and 14 bits of resolution.), a mini computer (Raspberry, for example). This combination allows to install an embedded operating system (e.g. Linux) that can run the necessary software for the Data Acquisition (DAQ), like the ABCD distributed DAQ.

Our contribution will be aimed to show a comprehensive characterization of the two detection systems, a medium size CLLB scintillation detector, and a large plastic scintillator, EJ-276, in order to assess their potential use in a UAV-based radiation monitoring system.

Keywords - UAV, neutron-gamma, scintillation detectors

\section{INTRODUCTION}

$\mathrm{R}$ ADIOACTIVE and nuclear materials may compose a threat to public health and homeland security in the form of terrorism menaces, lost orphan sources, nuclear accidents or radioactive contamination. Since the incident in March 2011 at the Fukushima Daiichi Nuclear Power Plant, a lot of effort has been devoted on the development of new unmanned ground and aerial vehicles (UGV and UAV respectively), equipped with radiation monitoring devices [1]. UAV are mainly used in accident scenarios, in areas of difficult access, and in cases where the radiation dose is expected to be too high for a manned survey. Frequently, the radiation monitoring capabilities of the detectors embedded on a UAV are suitable specifically for gamma-ray detection (dosimetry and spectrometry).

At present, the most common gamma ray detectors used are: Geiger-Müller (GM) tubes [2] , cadmium zinc telluride (CZT) detectors [3] [4] Martin et al. (2015, 2016); [5] Selivanova et al. (2019), and scintillators such as LaBr3:Ce [6] ; NaI:Tl [7] and CsI [8] . The GM tubes are used for dosimetry purposes, while, CZT detectors are adopted for identification of radionuclides (spectrometry). However, both kinds of devices have an inherently very low sensitivity, being lightweight (low electronic density, GM tubes) or small volume (max. $1 \mathrm{~cm} 3$, CZT). Small and medium-size scintillation detectors have been employed, they offer the possibility to perform dosimetry and spectrometry at the same time. In case of medium-size scintillators, the sensitivity is significantly increased with respect to the GM tubes and CZT detectors. However, the increase of size and weight of the UAV payload means more power consumption, thus less flight time or more powerful motors.

The Dragon (Drone for RAdiation detection of Gammas and Neutrons) project aims at designing, developing, and characterizing a UAV equipped with a detection system able to identify radioactive materials, spread over an area or located in a specific position. The Dragon project is being funded by the INFN_E commission of the Italian National Institute for Nuclear Physics (Istituto Nazionale di Fisica Nucleare, INFN).

The type of radioactive sources that will be detected are gamma emitters and special nuclear materials (SNM). In particular, the proposed technology incorporates an integrated thermal neutron and gamma ray detector, able to discriminate between the two kinds of particles and to perform goodresolution gamma spectroscopy. Neutron and gamma measurements are complementary: their combined power is expected to improve the system performances. SNMs (Highly Enriched Uranium and Plutonium) are difficult to detect, especially when masked or shielded. Gamma rays and neutrons emitted by SNMs have to be detected at the same time, in order to increase the sensitivity against natural background [9]. The Li-containing elpasolite scintillators (CLYC, CLLB, etc.) seem to be the right choice to fulfil the mentioned requirements: they exhibit excellent energy linearity; high light output, resulting in 
good energy resolution; have good thermal neutron detection efficiency, through the 6Li(n; ) $3 \mathrm{H}$ capture reaction. One aspect to take into account is that $\mathrm{La}-$ containing scintillators such as CLLB, CLLBC, $\mathrm{LaBr} 3, \mathrm{LaCl} 3$, etc. exhibit an intrinsic radioactive background, from naturally occurring 138La and from alpha decays from actinium (227Ac) contamination [10] [11] [12] [13] [14]

In the framework of the Dragon project, we present in this work the characterizations of a large plastic scintillator EJ276 and of a medium size CLLB (Cs2LiLaBr6:Ce) scintillation detector, in order to assess their potential use in a UAV-based radiation monitoring system. The parameters studied are: energy resolution, time resolution, neutron/gamma discrimination capability, high counting rate performance and the neutron in a high gamma flux. Fig. 1a shows a 3D rendering of the prototype of the detection system; it includes: the detector, a compact fast digitizer, a mini computer to analyse the data and a high voltage power supply. An anti-impact structure has been added in the mechanical design in order to protect all mentioned components. Fig. 1b shows a picture of the 3D-printed structure.

\section{EXPERIMENTAL OVERVIEW}

The detection system of the present study is made up of : a $3 \times 5$ " cylindrical EJ-276 plastic scintillator (from Eljen Technology, Texas)

a 2" x 2" cylindrical CLLB scintillation detector, purchased from Saint Gobain crystals.

The main physical properties of the plastic EJ-276 scintillator are: density 1.096 , light output (\% Anthracene) 56, scintillation efficiency $8600 \mathrm{ph} / \mathrm{MeV}$, wavelength of maximum emission $425 \mathrm{~nm}$, mean decay time $460 \mathrm{~ns}$ for neutron and $270 \mathrm{~ns}$ for gamma.

The plastic scintillator is coupled with a PMT(H6559) Hamamatsu photomultiplier.

The main physical properties of the CLLB scintillation detector are: density $4.2 \mathrm{~g} / \mathrm{cm} 3$, light output $40000 \mathrm{ph} / \mathrm{MeV}$, hygroscopic, wavelength of maximum emission $420 \mathrm{~nm}$ and decay time, according to the manufacturer, $180 \mathrm{~ns}$ (50\% for gamma and $61 \%$ for $\mathrm{n}$ ) and $1080 \mathrm{~ns}$ (50\% for gamma and $39 \%$ for $n$ ).

The scintillator was coupled to a 2" dia. PMT (R6231) Hamamatsu photomultiplier together with a voltage divider (AS20).

The PMT signals were acquired by two digitizers: a CAEN DT5725 fast digitizer (250MSamples/s, 14-bit ADC resolution and Digital Constant Fraction Discriminator, DCFD, embedded in the firmware for precise timing measurements) as reference for the CLLB and the plastic scintillator, and the RedPitaya STEMLAB 125-14 (125Ms/s, 14-bit ADC resolution, power consumption 2 A $5 \mathrm{~V}$ ) for the plastic one. The Red Pitaya is a portable light digitizer with two input channels, digitizer expected to be installed in the DRAGON project, due to its light weight and low power consumption. These digitizers allow to record the PMT current pulses as digitized waveforms, to perform subsequent off-line analysis. The installed firmware in the DT5725 is the Digital Pulse Processing for Charge Integration and Pulse Shape Discrimination, DPP-PSD, which can pre-process data determining the pulses timestamps and integrating them over two gates, for the so-called double integration PSD method Pino et al. (2014a).

Bias voltage was applied using a CAEN V6533 VME HV Power Supply Module. A CAEN V1718 VME-USB2.0 Bridge was used to control the HV power supply unit. A CAEN A3818 PCI Express CONET2 Controller installed on a PC was used to control the digitizer (by means of optical fibre connections using the CAEN CONET2 protocol). All parameters of the nuclear electronics, and also the data acquisition, were managed using a software called ABCD (Acquisition and Broadcast of Collected Data) [15] [16] [17] released as an open-source project.

To study the gamma response of the detectors, different calibration sources are used (137Cs, $22 \mathrm{Na}, 60 \mathrm{Co}, 133 \mathrm{Ba}, 57 \mathrm{Co}$ and $88 \mathrm{Y}$ ) with different activities between $200 \mathrm{kBq}$ and 300 $\mathrm{kBq}$.

To study the neutron and gamma response, a $252 \mathrm{Cf}$ source was used (neutron yield at the time of the experiments $\sim 5.2 \times 10^{4}$ $\left.\pm 20 \% \mathrm{~s}^{-1}\right)$.

\section{EXPERIMENTAL RESULTS}

One of the objectives of the DRAGoN project is to detect and identify SNM, and this can represent a challenging task taking into account that the SNM can be hidden or masked by a high gamma background, but with the simultaneous detection of neutrons and gamma-rays, it is possible to increase the sensibility. Therefore a very important feature that should be well known is the performance of the detection system in a high counting rate field. In the present work, the determination of the dead time of the detection system and the neutron detection response in a high gamma counting rate will be presented. Moreover, comparative results between different readouts with the plastic scintillator will be shown.

\section{A. High Counting Rate Tests}

In this kind of tests, in order to minimize the operators radiation exposure, the detector was fixed to a movable carriage on a guide rail, and its movements (change of position and speed) were controlled from a computer using the Linux CNC platform. The position of the gamma sources was fixed at one edge of the guide rail.

As far as the plastic scintillator system, the gamma sources used were ${ }^{60} \mathrm{Co},{ }^{22} \mathrm{Na},{ }^{137} \mathrm{Cs}$ and ${ }^{133} \mathrm{Ba}$, while for the CLLB the source was a $185 \mathrm{MBq}{ }^{60} \mathrm{Co}$ gamma source.

\section{1) Plastic Scintillator EJ-276}

A comparison of the digitizer performance can be seen in Fig. 1 , where the red line represents an equivalent behaviour between both digitizers. The dead time of the RedPitaya was determined for each measurement using the CAEN DT5725 digitizer as a reference. The experimental results (blue line in Fig.1) show that when the counting rate is increasing the RedPitaya performance decreases since it can be seen that there is a logarithmic growth of the dead time. We can conclude that 
for a counting rate equal to $3 \mathrm{kcps}$ the rate of lost events is about $15 \%$.

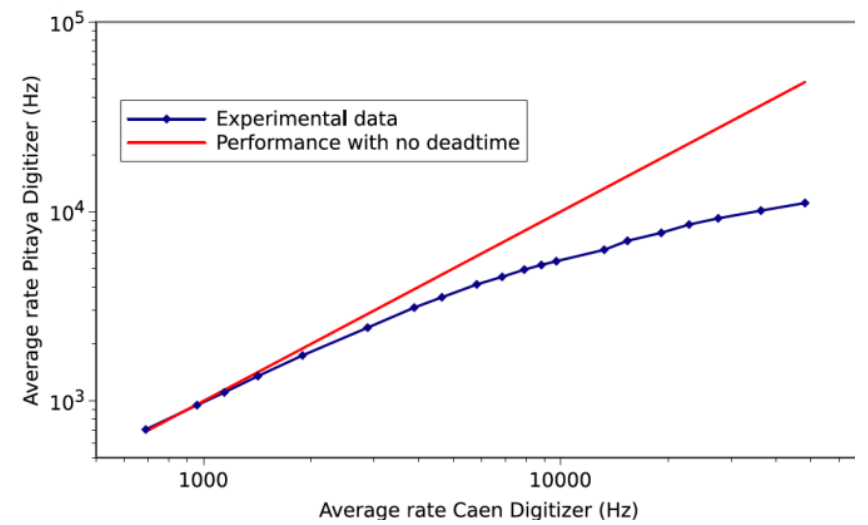

Figure 1: Performance of the RedPitaya digitizer as a function of the counting rate. The average rate of the Red Pitaya digitizer respect to the CAEN digitizers is presented as a function of the counting rate.

\section{2) CLLB detection system}

In this case, several gamma energy spectra for a determined counting rate were acquired (each corresponding to a sourcedetector distance). Only on-line analysis was employed. From the spectra, the relative position of the $1332 \mathrm{keV}$ full energy peak and the energy resolution (at the same energy) were studied as a function of the counting rate. The results are given in Fig. 2. It can be concluded that with counting rates up to about $40 \mathrm{kcps}$ the detector gives a response with a gain shift lower than $4.5 \%$, and with an energy resolution @ $1332 \mathrm{keV}$ lower than about $5 \%$.

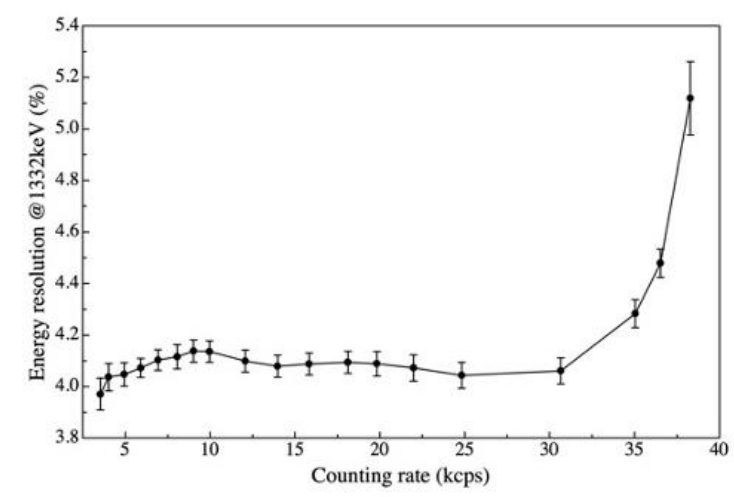

Figure 2: Energy resolution as a function of the counting rate for the CLLB detector.

\section{B. Neutron detection response in a high gamma counting rate}

Neutron detection response in a high gamma field is important taking into account the DRAGON project field of application. In the case of high gamma filed gamma events can be detected and determined as fake neutrons counts, giving unwanted false alarms. In these tests a $252 \mathrm{Cf}$ source is used together with a 60Co gamma source.

The PSP paramater used in the neutron/gamma discrimination for the plastic scintillator is defined as $\mathrm{Q}_{\text {tail }} / \mathrm{Q}_{\text {short }}$, while for the CLLB detector is defined as $\mathrm{Q}_{\text {shor }} \mathrm{t} / \mathrm{Q}_{\text {long. }}$ ).

\section{1) Plastic Scintillator EJ-276}

In these tests, a 1 hour ${ }^{252} \mathrm{Cf}$ source measurement has been performed as a reference for the neutron counts. In particular, the region with the following limits have been considered as "neutron region": E1=0.1 MeV, E2=0.57 MeV, E3=2.5 MeV, PSDmin $=0.2$ and PSDmax $=0.614$, and the slope is a fourdegree polynomial function, see (1).

$$
f(x)=1.08756-6.74552 x+21.3637 x^{2}+
$$$$
31.0788 x^{3}+16.9939 x^{4} \quad(1)
$$

The neutron region is clearly indicated by the red line in the Fig. 3 of the 2D plot, PSP parameter for the plastic scintillator vs light output.

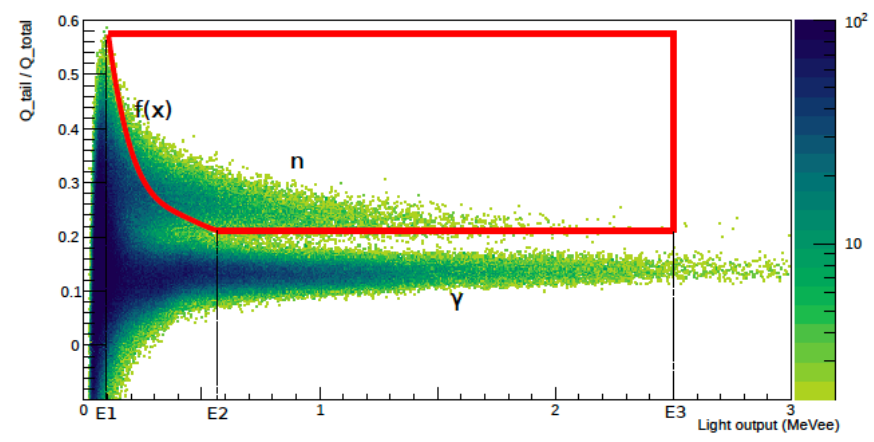

Figure 3: 2D-PSD of a 252Cf source, with the neutron contoured region (red line). Using the Ej-2763"x5", the RedPitaya digitizer and the PMT model H6559.

Once defined the neutron region, the high gamma rate measurements were performed placing a 60Co source next to the $252 \mathrm{Cf}$ source where different gamma rates were obtained by approaching the gamma source to the detector, each measurement has a time of around $10 \mathrm{~min}$. The number of events in the contoured region was determined as a function of the mean rate. The results with respect to the $252 \mathrm{Cf}$ source measurement can be seen in Fig. 4. Up to $3-4 \mathrm{kHz}$, no increase in the neutron region can be seen since there is not a significant increase in the neutron contoured region.

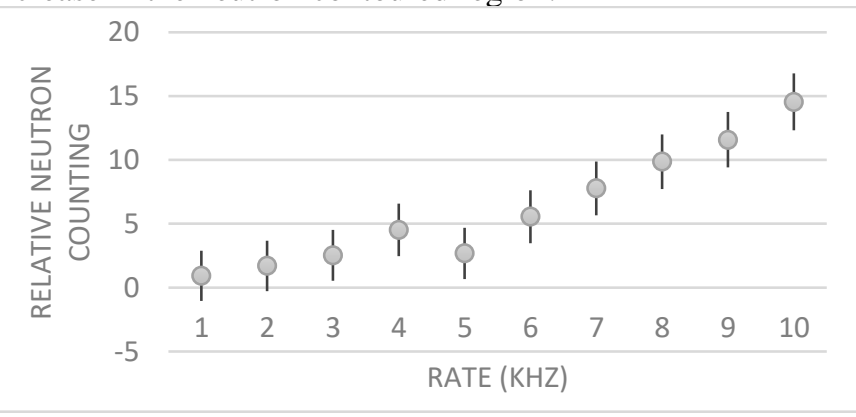

Figure 4: The neutron counting rate in a high gamma field is reported as a function of the counting rate.

\section{2) CLLB Detector}

In the CLLB detection system, the results of high gamma intense field is shown in Fig.5, where the 2D plot, PSP parameter for the CLLB versus light output is reported. 
From Fig.5 it is clear that the high gamma events are in the region of smallest PSP respect to the neutron region (red line in Fig.5).

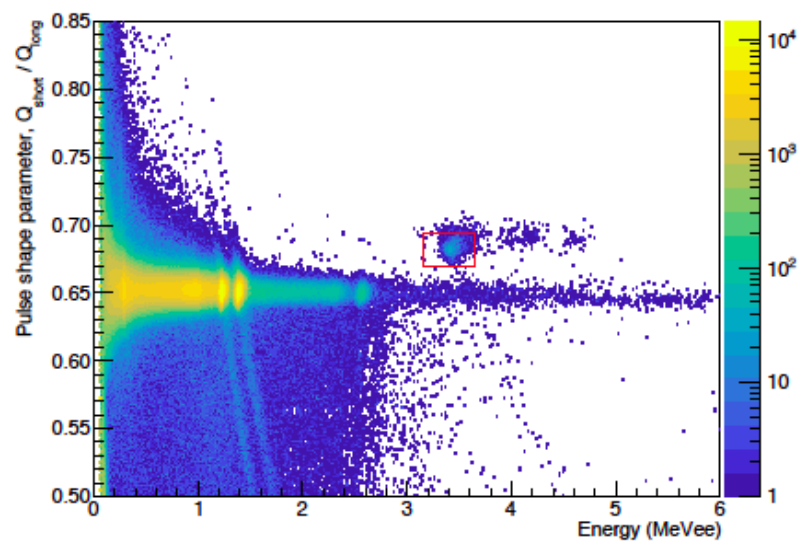

Figure 5: PSP parameter vs light output foe the CCLB detector in a $15 \mathrm{kHz}$ gamma rate.

For this reason, a higher gamma flux can be resolved with this detector, in fact up to gamma rate of $30 \mathrm{kcps}$, the neutron counts lost are lower than $10 \%$. The losses are mainly due to pile-up and coincidence summing effects, which become more important as gamma rate increases. This is clearly seen in Fig. 6 , where the relative neutron counting rate is reported as a function of the count rate.

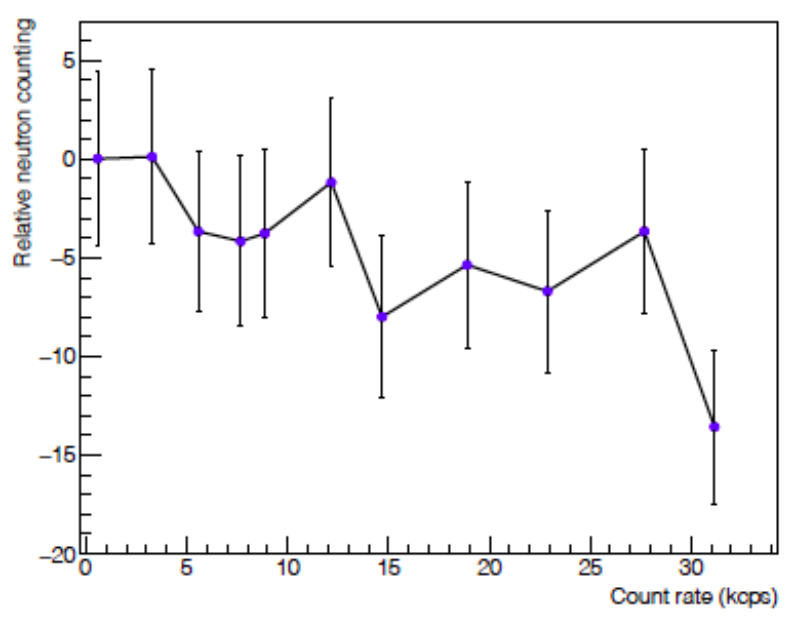

Figure 6: The relative neutron counting as a function of the total counting rate.

\section{3) Comparative results of Red Pitaya and CAEN digitizer} with plastic scintillator EJ276.

The Red Pitaya is a portable light digitizer, expected to be installed in the DRAGON project, due to its light weight and low power consumption. An important characteristic to be checked is its intrinsic capabilities to discriminate the neutron and gamma events in the case of the plastic scintillator.

In order to characterize the discrimination capability of the detector, the Figure of Merit (FoM), defined as , FoM = $\frac{\Delta}{\left(\delta_{n}+\delta_{\gamma}\right)}$ was computed by analyzing the PSP distribution[18] , $\Delta$ is the difference between the two mean values of the neutron and gamma distributions and $\left(\delta_{n}+\delta_{\gamma}\right)$ is the sum of the neutron and gamma Full Width at Half Maximum (FWHM). The results are shown in Fig.7. The resulted FoM is greater with the CAEN digitizer as expected, but, the results with Red Pitaya are still very good, obtaining a FoM of about 1.1 at $1 \mathrm{MeV}$.

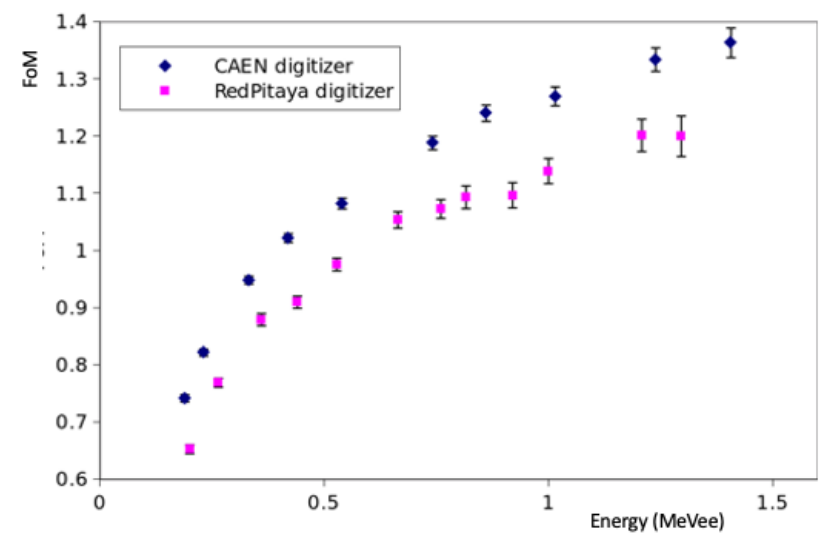

Figure 7: The FoM vs Energy for the plastic scintillator EJ276.

\section{CONCLUSION}

In this paper, we presented the DRAGON project and the detection system performances in high gamma rate, in terms of dead time for the plastic scintillator system and energy resolution for the CLLB system. Moreover, the relative neutron counting rate in a high gamma files is presented for both detection solution.

As a result, for the plastic scintillator and the RedPitaya, up to $3 \mathrm{kHz}$ (about 3 time more the natural radioactivity), the rate of lost events is about $15 \%$ and there is no a significant increase in the neutron contoured region. As far as the CLLB detector and the CAEN digitizer, higher counting rates can be considered, since, with counting rates up to about $40 \mathrm{kcps}$ the detector gives a response with a gain shift lower than $4.5 \%$, and with an energy resolution@1332keV lower than about 5\%. Moreover, up to gamma rate of $30 \mathrm{kcps}$, the neutron counts lost are lower than $10 \%$. For the CLLB the performances with RedPitaya are ongoing, a slightly change in the total counting rate is expected but no increasing in the neutron counting rate is expected.

The use of the Red Pitaya has bene demonstrated to be a very good choice in this kind of application, where the power consumption is a key parameter to be considered, given a very satisfactory results in terms of discrimination capabilities between gamma and neutron.

The next steps will be the study of the detection system performances on the UAV itself with a whole characterization in terms of efficiency, altitude and velocity.

\section{ACKNOWLEDGEMENTS}

This work is supported by the INFN_E commission of the Italian National Institute for Nuclear Physics through the Dragon (Drone for RAdiation detection of Gammas and Neutrons) project.

\section{REFERENCES}

[1] D. Connor, P.G. Martin, T.B. Scott, "Airborne radiation mapping: Overview and application of current and future aerial systems" International Journal of Remote Sensing 2016;37(24):5953-87, 10.1080/01431161.2016.1252474. 
[2] K. Kurvinen, P. Smolander, R. Pöllänen, S. Kuukankorpi, M. Kettunen , J. Lyytinen, "Design of a radiation surveillance unit for an unmanned aerial vehicle" Journal of Environmental Radioactivity 2005;81(1):1-10, 10.1016/j.jenvrad.433 2004.10.009.

[3] P. Martin, O. Payton, J. Fardoulis, D. Richards, T. Scott, "The use of unmanned aerial systems for the mapping of legacy uranium mines" Journal of Environmental Radioactivity 2015;143:135-40, 10.1016/j.jenvrad.2015.437 02.004.

[4] P. Martin, O. Payton, J. Fardoulis, D. Richards, Y. Yamashiki, T. Scott, "Low altitude unmanned aerial vehicle for characterising remediation effectiveness following the FDNPP accident" Journal of Environmental Radioactivity 441 2016;151:58-63, 10.1016/j.jenvrad.2015.09.007.

[5] A. Selivanova, J. Hulka, T. Vrba, I. Cešpírová, "Efficiency calibration of a CZT detector and MDA determination for post accidental unmanned aerial vehicle dosimetry" Applied Radiation and Isotopes 2019;154:108879, doi:10.1016/j.apradiso.2019.108879.

[6] Y. Sanada, T. Torii, "Aerial radiation monitoring around the Fukushima Dai-ichi nuclear power plant using an unmanned helicopter", Journal of Environmental Radioactivity 2015;139:294-9, 10.1016/j.jenvrad.2014.06.027.

[7] K. Kurvinen, P. Smolander, R. Pöllänen, S. Kuukankorpi, M. Kettunen, J. Lyytinen, "Design of a radiation surveillance unit for an unmanned aerial vehicle", Journal of Environmental Radioactivity 2005;81(1):1-10, 10.1016/j.jenvrad.2004.10.009.

[8] R. Pöllänen, H. Toivonen, K. Peräjärvi, T. Karhunen, T. Ilander, J. Lehtinen, K. Rintala, T. Katajainen, J. Niemelä, M. Juusela, "Radiation surveillance using an unmanned aerial vehicle", Applied Radiation and Isotopes 2009;67(2):340-4, 10.1016/j.apradiso.2008.10.008.

[9] D. Cester, G. Nebbia, L. Stevanato, G. Viesti, F. Neri, S. Petrucci, et al., "Special nuclear material detection with a mobile multi-detector system", Nuclear Instruments and Methods in Physics Research Section A: Accelerators, Spectrometers, Detectors and Associated Equipment 2012;663(1):55-63, 10.1016/j.nima.2011.10.011.

[10] J. Hartwell, R. Gehrke, "Observations on the background spectra of four $\mathrm{LaCl} 3(\mathrm{Ce})$ scintillation detectors", Applied Radiation and Isotopes 2005;63(2):223-8, 10.1016/j.apradiso.2005.02.009.

[11] W. Kernan, "Self-activity in lanthanum halides", IEEE Transactions on Nuclear Science 2006;53(1):395-400, 10.1109/TNS.2006.869849.

[12] R.S. Woolf,B.F. Phlips, W.A.Wulf, "Characterization of the internal background for thermal and fast neutron detection with CLLB", Nuclear Instruments and Methods in Physics Research Section A: Accelerators, Spectrometers,Detectors and Associated Equipment 2016;838:147-53, 10.1016/j.nima.2016.09.013.

[13] G. Hull, F. Camera, G. Colombi, M. Josselin, B. Million, N. Blasi, "Detection properties and internal activity of newly developed Lacontaining scintillator crystals", Nuclear Instruments and Methods in Physics Research Section A: Accelerators, Spectrometers, Detectors and Associated Equipment 2019;925:70-5, doi:10.1016/j.nima.2019.01.094.

[14] R.S. Woolf, E.A. Wulf , B.F. Phlips, P. Chowdhury, E.G. Jackson, "Identification of internal radioactive contaminants in elpasolites (CLYC, CLLB, CLLBC) and other inorganic scintillators", Nuclear Instruments and Methods in Physics Research Section A: Accelerators, Spectrometers, Detectors and Associated Equipment 2020;954:161228, 10.1016/j.nima.2018.09.063.

[15] C. L.,Fontana, M. Lunardon, F. E. Pino, L. Stevanato, A. Carnera, C. Sada, F. Soramel, S.Moretto, (2018). "A distributed data acquisition system for nuclear detectors". International Journal of Modern Physics: Conference Series, 48 , 1860118., 10.1142/S2010194518601187.

[16] C.L. Fontana, M. Lunardon, F.E. Pino, L. Stevanato, S. Moretto, (2019) "Resource sharing in nuclear physics laboratory classes: A distributed data acquisition system for experiments with shared resources and data management." In 25th international conference on the application of accelerators in research and industry (p. 050024). Texas, doi:10.1063/1.5127716.

[17] C. L. Fontana, M. Lunardon, F. E. Pino, L. Stevanato, A. Carnera, C. Sada, F. Soramel, S.Moretto, (2017b). "A distributed data acquisition system for signal digitizers with on-line analysis capabilities". In 2017 IEEE Nuclear Science Symposium and Medical Imagi

[18] D. Cester, G.Nebbia, L. Stevanato, F.E. Pino, G. Viesti, (2014) "Experimental tests of the new plastic scintillator with pulse shape discrimination capabilities EJ-299-33". Nuclear Instruments and Methods in Physics Research Section A: Accelerators, Spectrometers, Detectors and Associated Equipment, 735 , 202-206. doi:10.1016/j.nima.2013.09.031. 